\title{
Calculus-enhanced energy-first curriculum for introductory physics improves student performance locally and in downstream courses
}

\author{
Sarah E. LeGresley, Jennifer A. Delgado, Christopher R. Bruner, \\ Michael J. Murray, and Christopher J. Fischer" \\ Department of Physics and Astronomy, University of Kansas, 1251 Wescoe Hall Drive, 1082 Malott Hall \\ Lawrence, Kansas 66045, USA
}

(Received 29 March 2019; published 13 September 2019)

\begin{abstract}
Here we demonstrate the benefits of a new curriculum for introductory calculus-based physics that motivates classical mechanics using a modified version of Hamiltonian mechanics. This curriculum shifts the initial focus of instruction away from forces and the associated vector mathematics, which are known to be problematic for students, to the scalar quantity energy, which is more closely aligned with their previously established intuition, and associated differential and integral calculus. We show that implementation of this calculus-enhanced "energy-first" curriculum resulted in higher normalized gains on the Force Concept Inventory exam for all students and improved performance in downstream engineering courses for students with lower ACT math scores. In other words, the downstream benefits were largest for students with lower math abilities who also pose a larger retention risk. This new curriculum thus has the potential to improve student retention by specifically helping the students who need help the most, including traditionally underserved populations who often have weaker mathematics preparation. We propose future work to investigate whether this new curriculum has lowered the math transference barrier to learning in introductory physics, resulting concomitantly in improvements in student learning of classical mechanics and in student fluency with applied mathematics.
\end{abstract}

DOI: 10.1103/PhysRevPhysEducRes.15.020126

\section{INTRODUCTION}

Nearly all undergraduate science, technology, engineering, and mathematics (STEM) degree programs require courses in introductory physics since the content of these courses is considered foundational to those disciplines. It is therefore not surprising that continually increasing enrollments in STEM degree programs [1] have only intensified the need for physics education research (PER) into improving instruction in these courses. The clear conclusion from numerous PER studies over the past two decades [2-4] is that student learning and overall student performance in both algebra-based and calculus-based introductory physics courses can be improved by incorporating active learning techniques such as Peer Instruction $[5,6]$ or inquiry-based instruction [7,8] into the classroom. Here we demonstrate that further improvement in student learning in calculus-based introductory physics may be obtained

\footnotetext{
Corresponding author. shark@ku.edu

Published by the American Physical Society under the terms of the Creative Commons Attribution 4.0 International license. Further distribution of this work must maintain attribution to the author(s) and the published article's title, journal citation, and DOI.
}

by supplementing these modifications of content delivery with a rearrangement of the content itself.

The goal of this project was to develop a new curriculum for introductory calculus-based classical mechanics and thermodynamics that would (i) improve student learning in the course, in terms of both a conceptual understanding of content and an ability to apply that content in quantitative problem solving, and (ii) task students with using calculus more regularly when solving problems. We found that we could address both outcomes of our goal by developing classical mechanics starting with the concept of energy rather than the concept of force; in this way, we are mimicking the instruction of classical mechanics in upper-level courses (e.g., Hamiltonian and Lagrangian mechanics). Indeed, our new curriculum tasks students initially with differentiating and integrating equations for energy to describe the mechanics of systems and delays the introduction of forces and Newton's laws until after students have gained proficiency with the concepts of classical mechanics using energy-based approaches. The increased emphasis on applied calculus continues throughout the course including, for example, tasking students with differentiating and integrating equations for entropy to determine the equilibrium conditions of systems.

We further hoped that placing the initial focus of introductory physics on the concept of energy, and more generally on the concept of a conservation law, rather than 
on the concept of force would enable us to create for students consistent and effective scaffolding for solving a wide variety of problems [9] and thereby help students understand course content better. We were also encouraged to pursue this "energy-first" approach by the results of previous studies documenting student difficulty with the vector mathematics required to use forces correctly [10-13] and those indicating that students' intuitions about the concept of energy - from everyday experience or previous course work (e.g., in chemistry) - are more correctly aligned with the concept of energy within the context of physics than are their intuitions about force $[14,15]$. We also suspected that students would be more proficient using calculus to solve mechanics problems involving a scalar quantity (energy) rather than a vector quantity (force) [11-13].

We use two different assessments of whether these modifications of curriculum improved student learning in introductory physics, in terms of both a better conceptual understanding of course content and an improved ability to apply physics principles or methods when solving quantitative problems. We first compared the normalized gain on the standardized Force Concept Inventory (FCI) exam [16] for students completing our new curriculum [17] and students completing a traditional "force-first" curriculum [18]. However, since the FCI exam is focused on only a subset of the concepts covered in introductory physics and includes no quantitative questions, it is not necessarily a good metric of student fluency with introductory physics content and application. Thus we also assessed the impact of our new curriculum on student performance in downstream courses that list introductory physics as a prerequisite. Both analyses are consistent with this new curriculum improving student learning, especially for students with initially poor mathematics skills, as assessed using ACT math score as a proxy for mathematics ability. We discuss possible broader implications for this result and propose future work to discriminate them further.

\section{COMPARED COURSES}

Prior to the 2013-2014 academic year, the Department of Physics and Astronomy at the University of Kansas (KU) offered a single, three credit hour course for the first semester of calculus-based introductory physics. This course, PHSX 211 (General Physics I), was required by all natural science and engineering degree programs at KU. Beginning with the 2013-2104 academic year, the Department of Physics and Astronomy has offered two versions of the first semester of calculus-based introductory physics: PHSX 210 (General Physics I for Engineers) and PHSX 211. PHSX 210 is a three credit hour course, as PHSX 211 was previously, and the new version of PHSX 211 is a four credit hour course; the extra 50 min of class time in PHSX 211 is split equally between two of the three weekly class periods. The split into two physics courses was primarily done for institutional reasons to support the School of Engineering and not for the purposes of this study. Nevertheless, we have subsequently used this course split to study the impacts of changes to the PHSX 211 curriculum. Because of a variety of logistical reasons occurring each semester, not all KU School of Engineering (SOE) students are able to enroll in PHSX 210, resulting in enrollment of some SOE students in PHSX 211.

Figure 1 shows the distributions of ACT math scores for students in PHSX 210 and PHSX 211 from the fall semester of 2015 through the spring semester of 2018. The distributions for both PHSX 210 and PHSX 211 are very similar. Indeed, the only differences appear to be a higher fraction of lower $(<25)$ ACT math score students in PHSX 211 and a higher fraction of higher (>28) ACT math score students in PHSX 210. Since the average DFW rates over this same time period are also similar for the two classes $-13.9 \pm 1.3$ for PHSX 210 and $15.2 \pm 0.6$ for PHSX 211-the differences in student performance discussed here cannot be attributed to sample bias associated with the potential withdrawal of more lower performing students from PHSX 211 than from PHSX 210.

Neither PHSX 210 nor PHSX 211 is a purely lecture-based course, rather each employs student-centered active learning techniques $[5,6]$. Indeed, our department had transitioned all introductory courses to a hybrid format before the creation of PHSX 210. Before coming to class, students are tasked with reading the section of the textbook to be covered that day and/ or watching an online course video on the topic. Typically, no more than 15-20 min of class time are devoted to formal presentation by the professor. These presentations take the form of a conceptual quiz for the entire class (answered using an electronic response system or voting cards) or a minilecture on some aspects of that day's topic. During the majority of class time, students work together (in groups of three or four) to solve multiple problems on worksheets, with the professor

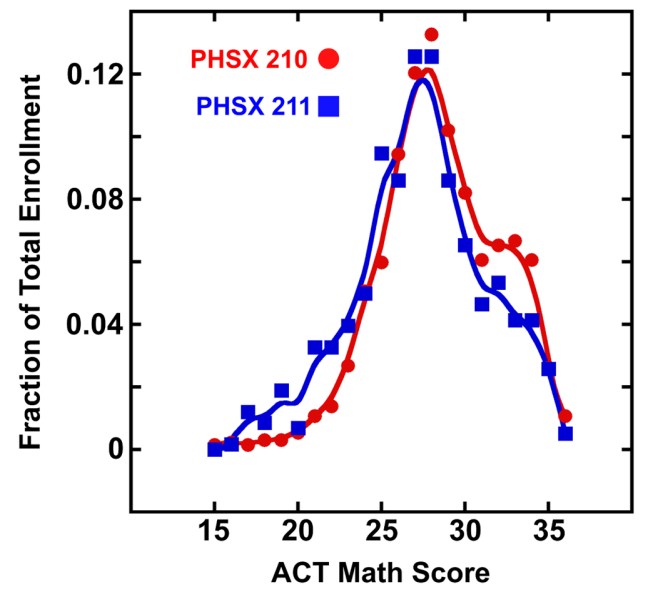

FIG. 1. The distribution of ACT math scores for students enrolled in PHSX 210 (red circles) and PHSX 211 (blue squares) between the fall semester of 2015 and the spring semester of 2018. The means and standard errors of the two distributions are $30.33 \pm 0.14$ for PHSX 210 and $29.4 \pm 0.3$ for PHSX 211. The solid lines are interpolations of the data meant to guide the eye. 
and undergraduate learning assistants available to help; the ratio of undergraduate learning assistants to students is the same for both PHSX 210 and PHSX 211. This approach provides students an opportunity to learn from each other as they develop the problem solving and critical thinking skills required to be successful in the course. After class, the students solve homework problems (administered and graded online) and are well prepared for these assignments after the practice they obtained before and during class. Although the content delivery in PHSX 210 and PHSX 211 is the same, within the variation expected for different instructors (in this study, these courses were taught by four different faculty members, all of whom are tenured full professors), the organization of the content in the two courses is different. While PHSX 210 follows a traditional curriculum in which the concept of force is introduced first in the discussion of classical mechanics [18], the curriculum in PHSX 211 develops classical mechanics using the concept of energy [17].

\section{CALCULUS-ENHANCED ENERGY-FIRST CURRICULUM}

Starting in the spring semester of 2015 we have used a new curriculum for PHSX 211 that develops classical mechanics using the concepts of energy and energy conservation before introducing the concept of force [17]. Our intention in structuring the curriculum in this way was to provide students a common conceptual and mathematical scaffold for solving a wide variety of problems. We further hoped that maintaining a common problem-solving strategy over as expansive a range of topics as possible would help students recognize the commonalities of these topics as well as afford the students the opportunity to practice and refine their skills with the associated mathematics. Indeed, we hoped to help students understand the concepts that underpin the mathematical strategies and associated equations rather than the strategies themselves [9].

This energy-first approach is probably best described as introductory Hamiltonian mechanics as it teaches students to determine the equation of motion of a system (specifically, the acceleration of the system) through differentiation of the equation for the total energy $(E)$ of the system. If a system is isolated, the total energy of the system will be constant (i.e., the energy of the system will be conserved) and the first derivative of the energy will be zero. For example, consider a system that consists of a single object moving along in one dimension along the $x$ axis that has both kinetic energy $(K)$ and potential energy $(U)$. If this system is isolated, the total energy of the system is constant regardless of the position of the object:

$$
\begin{aligned}
\frac{d E}{d x} & =0 \rightarrow \frac{d}{d x}(K+U)=0 \\
\frac{d K}{d x}+\frac{d U}{d x} & =0 \rightarrow \frac{d}{d x}\left(\frac{1}{2} m v_{x}^{2}\right)+\frac{d U}{d x}=0 \\
m v_{x} \frac{d v_{x}}{d x}+\frac{d U}{d x} & =0 \rightarrow m v_{x} \frac{d v_{x}}{d t} \frac{d t}{d x}+\frac{d U}{d x}=0 \\
m v_{x} a_{x} \frac{1}{v_{x}}+\frac{d U}{d x} & =0 \\
m a_{x}+\frac{d U}{d x} & =0 \rightarrow a_{x}=-\frac{1}{m} \frac{d U}{d x}
\end{aligned}
$$

As expected, this final expression is merely a statement of Newton's 2nd law for conservative forces. When nonconservative forces are present, $d E / d x$ is equal to the net nonconservative force, which we introduce initially as an external source or sink of energy. An example comparing energy-first

\begin{tabular}{|c|c|c|c|c|}
\hline \multirow[b]{2}{*}{ Topic } & \multicolumn{4}{|c|}{ Principle mathematics } \\
\hline & Trigonometry & Vectors & Differentiation & Integration \\
\hline Kinematics & $210 / 211$ & $210 / 211$ & $210 / 211$ & 211 \\
\hline 1D and 2D motion & $210 / 211$ & $210 / 211$ & 211 & 211 \\
\hline Newton's 1st and 2nd laws & $210 / 211$ & $210 / 211$ & 211 & 211 \\
\hline Free-body diagrams & $210 / 211$ & $210 / 211$ & & \\
\hline Circular and rotational motion & $210 / 211$ & $210 / 211$ & 211 & 211 \\
\hline Energy conservation & $210 / 211$ & & 211 & $210 / 211$ \\
\hline Linear momentum & $210 / 211$ & $210 / 211$ & 211 & 211 \\
\hline Angular momentum & $210 / 211$ & $210 / 211$ & 211 & 211 \\
\hline Static equilibrium & $210 / 211$ & $210 / 211$ & & \\
\hline Fluid mechanics & & 210 & & \\
\hline Oscillatory motion & $210 / 211$ & & 211 & 211 \\
\hline Heat and 1st law & & & 211 & 211 \\
\hline Ideal gasses & $210 / 211$ & $210 / 211$ & & \\
\hline Entropy and 2nd law & & & 211 & 211 \\
\hline Thermodynamic equilibrium & & & 211 & 211 \\
\hline
\end{tabular}
and force-first solutions is provided in the Appendix.

In Table I we indicate the principle mathematical skills that students are tasked with using for each topic of the course.

TABLE I. Mathematics tasked to students for each topic in PHSX 210 [18] and PHSX 211 [17]. 
For example, students in PHSX 210 are routinely tasked with using trigonometry, vectors, and differentiation when studying kinematics. It is important to note that in Table I we are making a distinction between calculus-based explanations and derivations in the textbook (or in the lectures or other associated content) and exercises or problems assigned to students (on homework, exams, etc.) that require the use of calculus. The latter are the basis for Table I.

It is clear from Table I that our new energy-first curriculum relies more heavily on both differential and integral calculus than the traditional force-first curriculum. For example, PHSX 211 students are tasked with using calculus routinely when covering $1 \mathrm{D}$ and 2D translational motion, circular motion, rotational motion, and oscillatory motion, whereas PHSX 210 students are not. We hoped that this repeated use of calculus through the curriculum would help students improve their fluency with applying calculus to solving physics problems. For example, as shown above, we feel that the energy-first curriculum clearly aligns the mathematical operation of taking a derivative with the concept of a conservation law and thereby allows students to move beyond a simple, operational understanding of mathematics to a more abstract understanding of mathematics as a tool for describing the world around us. Indeed, we believe this curriculum empowers students to derive their own equations for describing systems and solving problems, rather than relying solely on equations derived by others.

In this energy-first curriculum [17], forces together with Newton's 1st and 2nd laws are introduced as an alternative approach to solving problems in classical mechanics and as a necessary tool for describing static equilibria; thus, the use of vectors is delayed until after classical mechanics has been developed using energy-based and calculus-based approaches. The presentation of these concepts follows the traditional pedagogy of free-body diagrams and force decomposition, but the students are taught first to associate forces with a change of energy through work, rather than with an acceleration using Newton's 2nd law. Similarly, Newton's 3rd law is introduced to distinguish energy exchanged within a system and energy exchanged outside of the system. The remainder of the curriculum consists of linear momentum, angular momentum, and thermodynamics. As shown in Table I, students following the energy-first curriculum are tasked with using differential and integral calculus during their study of thermodynamics; for example, students differentiate and integrate functions of entropy to describe the equilibrium states of systems. Relying continually on applied calculus in this way provides students a common mathematical framework connecting mechanics and thermodynamics that we felt was missing in traditional curricula (Table I).

\section{IMPROVED STUDENT PERFORMANCE ON CONCEPT INVENTORY EXAMS}

We first sought to assess our proposition that our new curriculum would improve learning in introductory physics by comparing the performances on the standardized FCI exam [16] (available from the PhysPort website [19]) of students who completed our new curriculum (PHSX 211 students) and students who completed the traditional curriculum (PHSX 210 students). Improvement in student learning can be quantified by a normalized change [20] between scores on exams taken at the beginning of the course (pretest) and scores on exams taken at the end of the course (post-test). The normalized change between a student's pretest and post-test scores, denoted as $c$, is calculated using

$c= \begin{cases}\frac{(\text { post-testscore })-(\text { pretestscore })}{100 \%-(\text { pretestscore })} & \text { post-test score }>\text { pretest score } \\ 0 & \text { post-test score }=\text { pretest score } . \\ \frac{\text { (post-testscore })-(\text { pretestscore })}{\text { pretestscore }} & \text { post-test score }<\text { pretest score }\end{cases}$

Students whose pretest and post-test scores are equal to each other and equal to either $100 \%$ or $0 \%$ are excluded from the calculation. The calculations presented here are for matched students only. The average normalized change $\left(c_{\text {avg }}\right)$ is the average of the normalized changes for all students in the course.

FCI exam data were collected for three semesters of PHSX 210 and three semesters of PHSX 211 during the 2016-2017 and 2017-2018 academic years; each of these semesters occurred after the implementation of our new curriculum in PHSX 211 in the spring semester of 2015. Since a positive correlation between mathematics ability and normalized FCI change has been widely reported [14,21-24], we aggregated and parsed the data by the ACT math scores of the students (i.e., we used ACT math score as a proxy for mathematics ability); in this analysis,

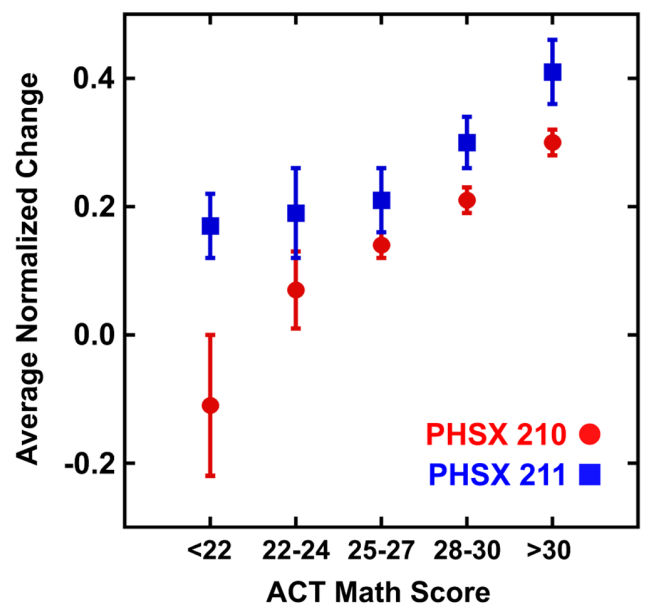

FIG. 2. The average normalized change for pretest and post-test FCI scores for students who completed PHSX 210 (red circles) or PHSX 211 (blue squares) sorted by ACT math scores. The uncertainty corresponds to the $68 \%$ confidence interval of the standard error. 
TABLE II. The average normalized change for pretest and post-test FCI scores for students who completed PHSX 210 or PHSX 211 sorted by ACT math scores. The uncertainty corresponds to the $68 \%$ confidence interval of the standard error.

\begin{tabular}{|c|c|c|c|c|c|}
\hline \multirow[b]{2}{*}{ ACT math score } & \multicolumn{2}{|c|}{ Sample size } & \multicolumn{2}{|c|}{$c_{\text {avg }}$} & \multirow[b]{2}{*}{$\Delta c_{\text {avg }}$} \\
\hline & PHSX 210 & PHSX 211 & PHSX 210 & PHSX 211 & \\
\hline$<22$ & 4 & 13 & $-0.11 \pm 0.11$ & $0.17 \pm 0.05$ & $0.28 \pm 0.12$ \\
\hline 22 to 24 & 10 & 11 & $0.07 \pm 0.06$ & $0.19 \pm 0.07$ & $0.12 \pm 0.09$ \\
\hline 25 to 27 & 75 & 38 & $0.14 \pm 0.02$ & $0.21 \pm 0.05$ & $0.07 \pm 0.05$ \\
\hline 28 to 30 & 147 & 48 & $0.21 \pm 0.02$ & $0.30 \pm 0.04$ & $0.09 \pm 0.04$ \\
\hline$>30$ & 141 & 37 & $0.30 \pm 0.02$ & $0.41 \pm 0.05$ & $0.11 \pm 0.05$ \\
\hline
\end{tabular}

the ACT math score is the highest score achieved by the student from all attempts at the ACT exam. As shown in Fig. 2 and Table II, for each cohort of ACT math scores the average normalized change for students who completed PHSX 211 were consistently larger than those of students who completed PHSX 210. Thus, the results of this analysis are consistent with our new curriculum improving student learning of the concepts of Newtonian physics as assessed by the FCI exam.

\section{EFFECTS ON STUDENT PERFORMANCE IN DOWNSTREAM COURSES}

While normalized gain on the FCI exam is frequently used to assess student learning of (a subset of) concepts in introductory physics, it contains no quantitative questions. Therefore, in order to assess our proposition that our new curriculum would improve the ability of students to apply physics principles or methods when solving quantitative problems, we next examined how the completion of our new curriculum affected student performance in subsequent (i.e., downstream) courses taken at $\mathrm{KU}$ that list General Physics I (PHSX 210 or PHSX 211) as a prerequisite. We assume in this subsequent analysis that a course requiring introductory physics as a prerequisite will rely upon the content of introductory physics, including both an understanding of physics concepts and an ability to apply those concepts in quantitative problem solving. Assessing our curriculum in this way also allows us to expand definition of student learning of physics beyond the physics classroom and thereby also examine the retention of course content.

\section{A. Methods}

The principle analysis performed to assess student performance in downstream courses is the comparison of course grade distributions (as a function of student ACT math score) using a normalized $\chi^{2}$ calculated with [25]

$$
\chi^{2}=\sum_{i}^{n} \frac{\left(O_{i}-E_{i}\right)^{2}}{E_{i}} .
$$

When applying Eq. (2), we will consider one of the distributions to be the predicted distribution and the other to be the observed distribution. The variables $O_{i}$ and $E_{i}$ in Eq. (2) denote the observed and predicted number of students, respectively, in these distributions for the $i$ th pairing of course grade and ACT math score; the variable $n$ in Eq. (2) denotes the number of such pairings. A value of $\chi^{2}=0$ denotes that the predicted and observed distributions are identical whereas values of $\chi^{2}>n$ denote that the predicted and observed distributions are significantly different. Since Eq. (2) will return a value of infinity whenever $E_{i}=0$, we excluded in our analysis all elements for which $E_{i}=0$.

A reduced $\chi^{2}$, denoted as $\tilde{\chi}^{2}$, can be determined by dividing $\chi^{2}$ by the number of degrees of freedom in the analysis, and can subsequently be used to determine a probability that the two distributions are identical and, if desired, a $p$ value $[25,26]$. However, since we are typically comparing very different distributions, neither these $\tilde{\chi}^{2}$ nor the associated probabilities are metrics with the dynamic range necessary to compare the relative levels of agreement necessary for our analysis. Indeed, as shall be seen, we are often comparing very different distributions for which the probability of agreement is approximately zero.

\section{B. Improved performance in statics and dynamics}

The first downstream course we examined was Mechanical Engineering (ME) 211, Statics and Dynamics, as its only prerequisite is General Physics I (PHSX 210 or PHSX 211); the content of ME 211 includes the principles of statics, with particular attention to engineering applications and an introduction to mechanics of materials. We aggregated the grades earned in ME 211 since the summer semester of 2015 (immediately following the introduction of our new curriculum in PHSX 211 in the previous spring semester) through the spring semester of 2018 by students who had completed either PHSX 210 or PHSX 211; this corresponds to a total of six semesters of data. Students who transferred credit for an equivalent physics course from another institution or who received advanced placement credit for PHSX 211 or PHSX 210 were excluded from this analysis. 
The grades earned in ME 211 from the fall semester of 2015 through the spring semester of 2018 for students who had completed either PHSX 210 or PHSX 211 are shown in Fig. 3 and indicate a positive correlation between the grade earned in ME 211 and mathematics ability, again using ACT math score as a proxy for mathematics ability. This is consistent with previously published data showing positive correlations between mathematics ability and performance in physics courses [27-30] and engineering degree programs [31-33]. It is worth noting, of course, that although a positive correlation exists between ME 211 grade and ACT math score (Fig. 3), it is not immediately clear what function should be used to describe the relationship. Thus, in order to move toward a model-independent analysis, we next compared the PHSX 210 and PHSX 211 distributions by calculating a normalized $\chi^{2}$ using Eq. (2). In other words, we used Eq. (2) to assess whether the distribution of student grades earned by PHSX 211 students predicted (or agreed with) the distribution of grades earned by PHSX 210 students; thus, the PHSX 211 distribution was the expected distribution in Eq. (2) and the PHSX 210 distribution was the observed distribution in Eq. (2). This calculation yielded $\chi^{2}=227$ for $n=47$. Since $\chi^{2}>n$, this analysis suggests that the distribution of grades earned by PHSX 211 students in ME 211 differ significantly from the distribution of grades earned by PHSX 210 students in ME 211 [25]; we could also state this result as there being only a $0.35 \%$ probability that the distributions are identical [25].

The mean ACT math score and mean ME 211 grade for the PHSX 210 distribution are $28.8 \pm 0.2$ and $2.77 \pm 0.08$, respectively, and the mean ACT math score and mean ME 211 grade for the PHSX 211 distribution are $28.3 \pm 0.3$ and $2.8 \pm 0.1$, respectively; these uncertainties denote the $68 \%$ confidence of the standard error. While these mean values are similar, there is nevertheless a shift toward a

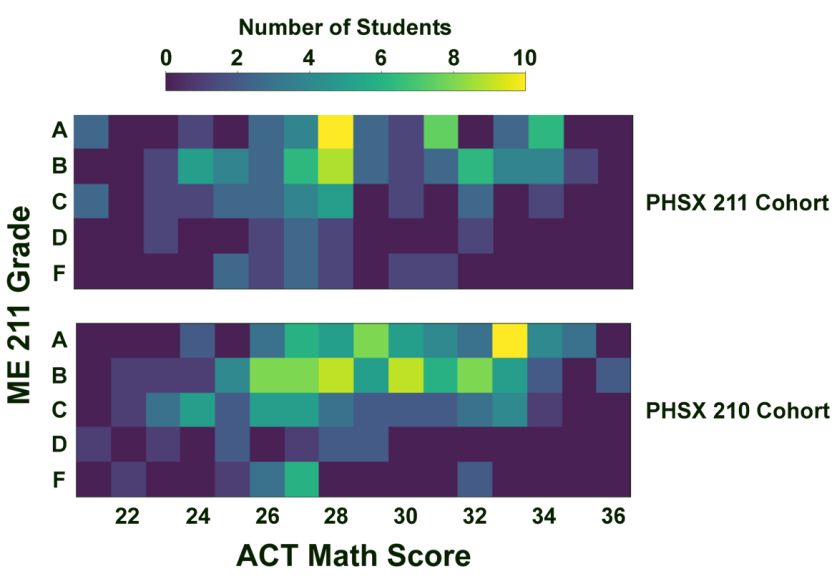

FIG. 3. The grades earned in ME 211 from the fall semester of 2015 through the spring semester of 2018 vs student's highest ACT math score for students who had completed PHSX 211 (top) or PHSX 210 (bottom). lower mean ACT math score and a higher mean ME 211 grade for the PHSX 211 cohort. We believe that this difference originates from the dependence of course grade on ACT math score being shallower for the PHSX 211 cohort than for the PHSX 210 cohort. In other words, if we equate ACT math score with math ability, math ability has less predicted power for grades earned in ME 211 for the PHSX 211 cohort than for the PHSX 210 cohort. In order to assess this proposition further, we decided to test the null hypothesis that ACT math score had no influence on grades earned in ME 211. The expected number of students earning each letter grade for each ACT math score was determined by multiplying the number of students with that ACT score by the grade distribution for ME 211, shown in Fig. 4; the product was rounded to the nearest whole number. This calculation for the PHSX 210 cohort of students completing ME 211 is shown in Fig. 5. These data illustrate that a smaller than expected number of the students with low ACT math scores earned an "A" or a "B" in ME 211 while, similarly, a smaller than expected number of students with high ACT math scores earned a "D" or "F" in ME 211. While the latter situation could be viewed as favorable, the former is almost certainly not favorable. To avoid our $\chi^{2}$ metric imparting a penalty for not giving enough lower grades to students with high ACT math scores, we decided to use the summed $\chi^{2}$ values for the grades of $\mathrm{A}$ and $\mathrm{B}$ in the quantitative assessment of downstream courses; the predicted and observed ACT math score distributions for students earning an A or B are shown in Fig. 5. We then compared these predicted and observed distributions by calculating a normalized $\chi^{2}$ using Eq. (2). In this analysis, a value of $\chi^{2}=0$ would be consistent with the null hypothesis and indicate that student ACT math score had no predictive power over student grade. As before, we also excluded all elements for which $E_{i}=0$ from this analysis. Applying Eq. (2) to the PHSX 210 cohort of students earning an A or B in ME 211 resulted in $\chi^{2}=33.0$. A similar analysis for the PHSX 211 cohort of students completing ME 211 resulted in a value of $\chi^{2}=22.6$. Since the agreement between the observed

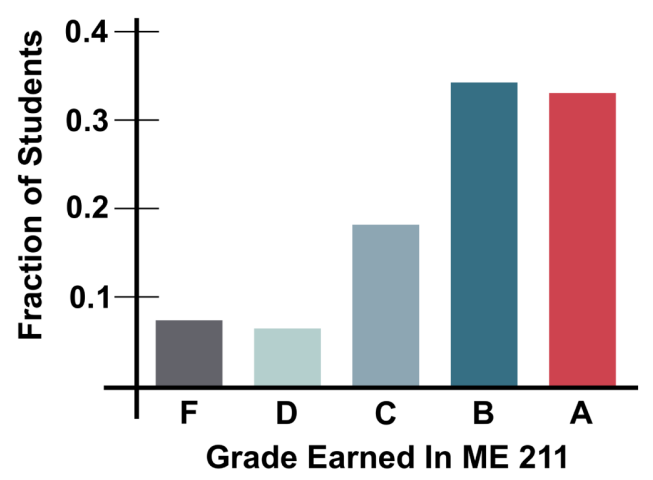

FIG. 4. The aggregated grade distribution for ME 211 between the fall semester of 2015 through the spring semester of 2018. 


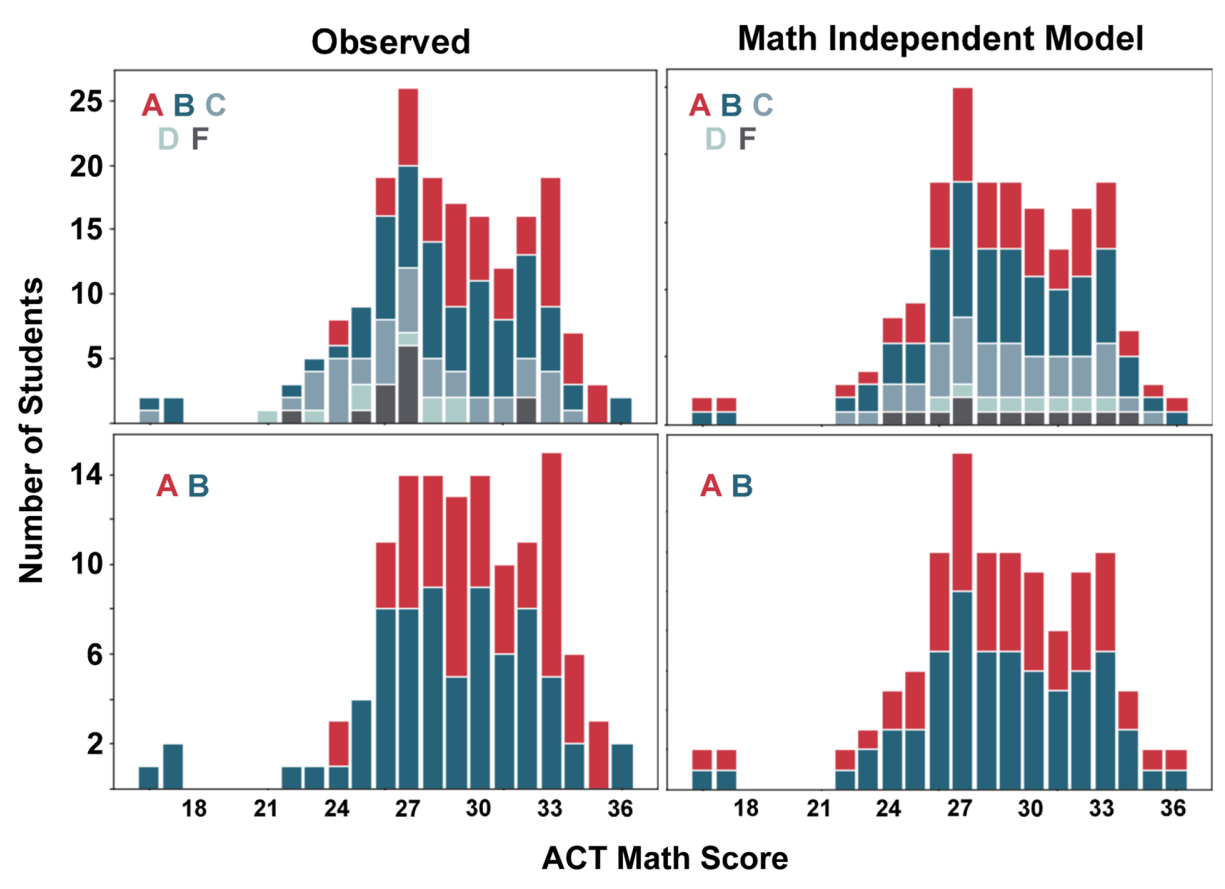

FIG. 5. The observed and predicted ACT math score distributions for students who completed PHSX 210. The left figures are the observed distributions and the right figures are the distributions expected if course grade is independent of ACT math score. The top shows the distributions for all course grades and the bottom rows are the distributions for students earning an A or a B.

and predicted distributions is better for the PHSX 211 cohort than for the PHSX 210 cohort, completion of PHSX 211 reduces the predictive power of ACT math scores on student grade in ME 211.

It is possible, of course, that the PHSX 211 cohort has a lower value of $\chi^{2}$ in ME 211 not because of the improvement in the performance of students with lower ACT math scores, but rather due to worse performance by students with higher ACT math scores (or some combination of both effects). In other words, it is possible that students with higher ACT math scores would perform better in ME 211 if they enrolled in PHSX 210 rather than PHSX 211. This possibility is not, however, supported by the data shown in Fig. 6. The distributions of grades earned in ME 211 by students with an ACT math score greater than 30 are very similar for the PHSX 210 and PHSX 211 cohorts; the mean grade earned by these students is 3.1 for the PHSX 210 cohort and 3.2 for the PHSX 211 cohort. In contrast, the distributions of grades earned in ME 211 by students with ACT math scores less than 25 show that these students are more likely to earn higher grades in ME 211 if they completed PHSX 211 (Fig. 6). This is reflected in the difference in the average grade earned by these students: 2.2 for the PHSX 210 cohort and 2.7 for the PHSX 211 cohort. Taken together, these data indicate that enrollment in PHSX 211 does not decrease performance in ME 211 for students with high ACT math scores, but instead increases the performance of students with lower ACT math scores. To put it another way, enrollment in PHSX 211 helps students with lower ACT math scores earn an A in ME 211 more so than enrollment in PHSX 210.

\section{Improved performance in other engineering and physics courses}

We next expanded our analysis of student performance to additional downstream courses in physics and engineering. In addition to being a prerequisite for ME 211, General Physics I (PHSX 210 or PHSX 211) is also a prerequisite for the following courses at $\mathrm{KU}$ :

Civil Engineering (CE) 301 Statics and Dynamics: The principles of statics, kinematics, and kinetics, with particular attention to engineering applications.

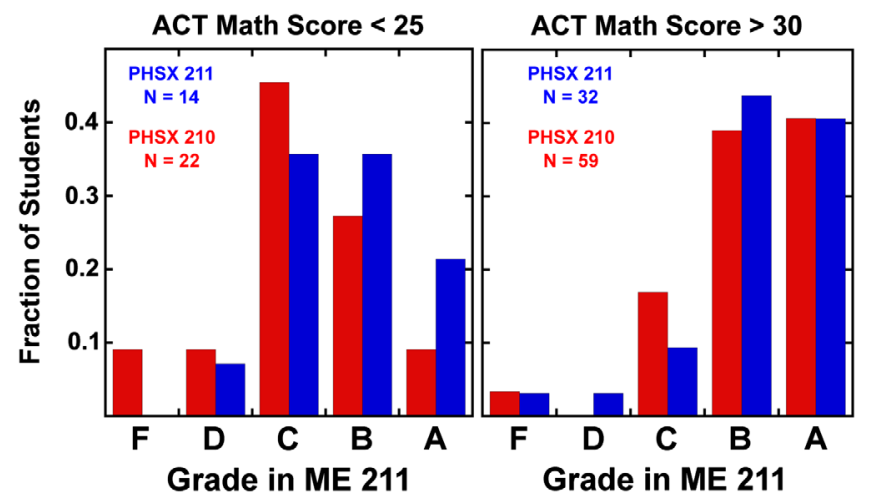

FIG. 6. The distribution of grades earned in ME 211 from the fall semester of 2015 through the spring semester of 2018 for students who completed PHSX 210 (red) or PHSX 211 (blue). Students with higher $(>30)$ ACT math scores within each cohort were equally likely to earn higher grades. In contrast, students with lower $(<25)$ ACT math scores were more likely to earn higher grades if they had been enrolled in PHSX 211. 
ME 312 Basic Engineering Thermodynamics: An introduction to the concepts of heat, work, the first and second laws of thermodynamics and equations of state. PHSX 212 General Physics II: Study of electricity and magnetism, waves, and sound.

The prerequisites and corequisites for these courses are shown in Fig. 7; in contrast to ME 211, these courses all have both General Physics I and Calculus II as prerequisites. As was done previously in our analysis of student performance ME 211 (Fig. 5), we calculated predicted distributions for students earning an A or a B in downstream courses and then used Eq. (2) to determine the summed $\chi^{2}$ values for students earning these grades. As shown in Fig. 8, prior enrollment in PHSX 211 resulted in lower predictive power of ACT math scores on student grades in CE 301 and ME 312. The summed $\chi^{2}$ values for PHSX 212 are nearly identical, suggesting that enrollment in PHSX 211 vs PHSX 210 has no effect on the predictive power of ACT math score on student grades in that course.

\section{Persistent and indirect improvements in student performance}

The analysis presented so far focused on downstream courses that are completed in the semester immediately following enrollment in General Physics I (PHSX 210 or PHSX 211). In order to examine the persistence of improved student performance associated with our new curriculum we examined the grades earned by the PHSX 210 and PHSX 211 cohorts in the following downstream course:

Chemical and Petroleum Engineering (C\&PE) 221 Chemical Engineering Thermodynamics: Fundamentals and applications of the first and second laws of thermodynamics with strong emphasis on material, energy, and entropy balances to solve engineering problems involving pure components.

The prerequisites for C\&PE 221 are shown in Fig. 9. It is important to emphasize that although C\&PE 221 lists General Physics I (PHSX 210 or PHSX 211) as a prerequisite, C\&PE 221 is taken one year after the completion of General Physics I, with an additional required thermodynamics course (Material and Energy Balances) taken during the intervening semester. We also included the following course to assess indirect effects of our new curriculum:

Aerospace Engineering (AE) 345 Fluid Dynamics: Study of fundamental aspects of fluid motions and basic principles of gas dynamics with application to the design and analysis of aircraft.

Although AE 345 is also typically taken in the semester following the completion of General Physics I (either PHSX 210 or PHSX 211), it does not list General Physics I as a prerequisite. AE 345 requires either CE

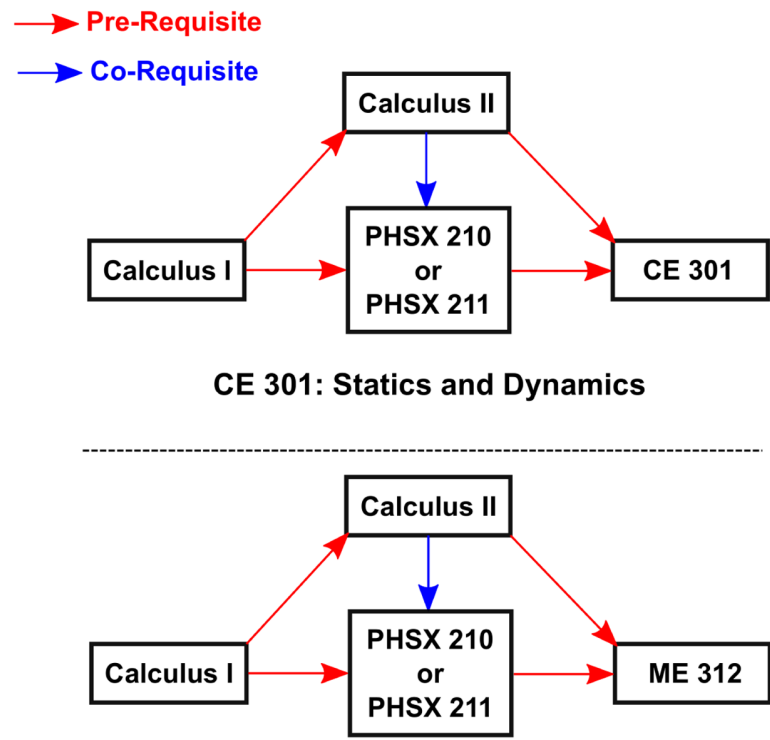

ME 312: Basic Engineering Thermodynamics

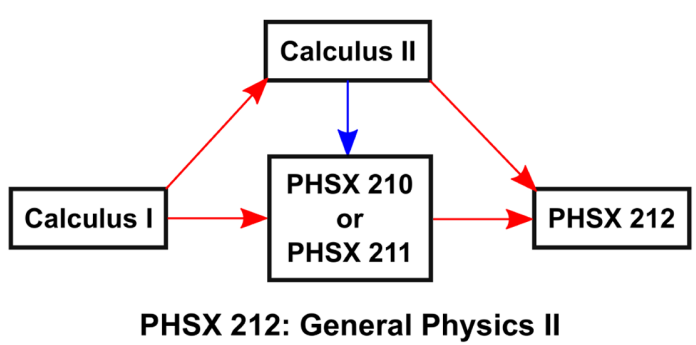

FIG. 7. The prerequisites and corequisites for CE 301 (Statics and Dynamics), ME 312 (Basic Engineering Thermodynamics), and PHSX 212 (General Physics II). All of these courses require Calculus II in addition to General Physics I.

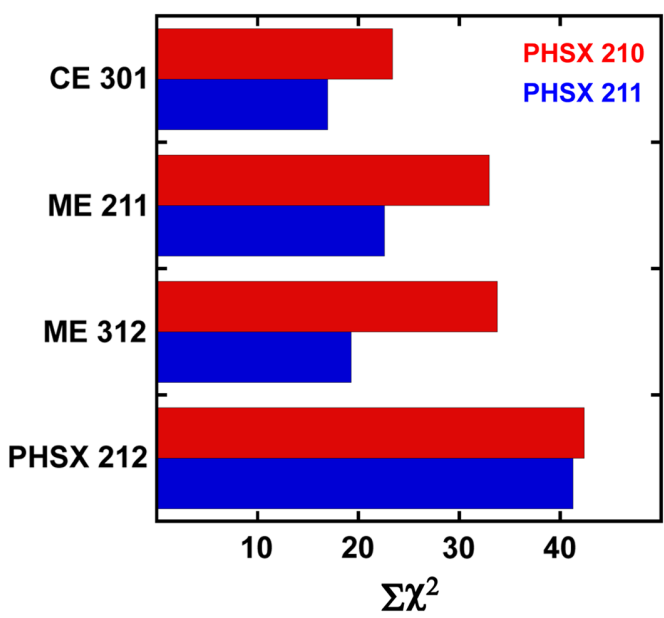

FIG. 8. Sum of $\chi^{2}$ for grades of A or B in downstream courses from the fall semester of 2015 through the spring semester of 2018 by students who had completed PHSX 210 (red) or PHSX 211 (blue). 


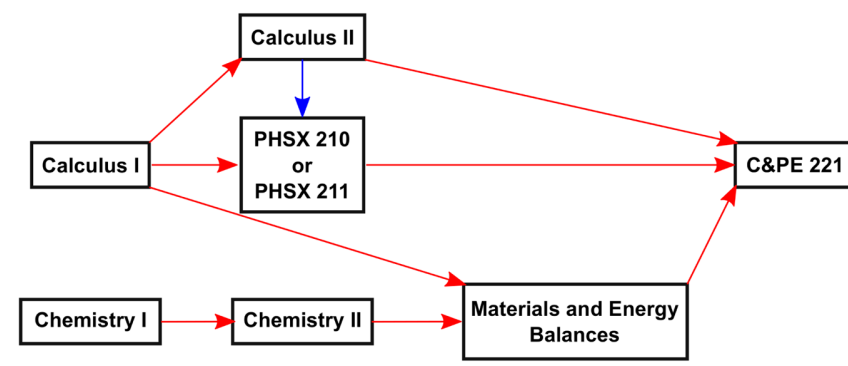

C\&PE 221: Chemical Engineering Thermodynamics
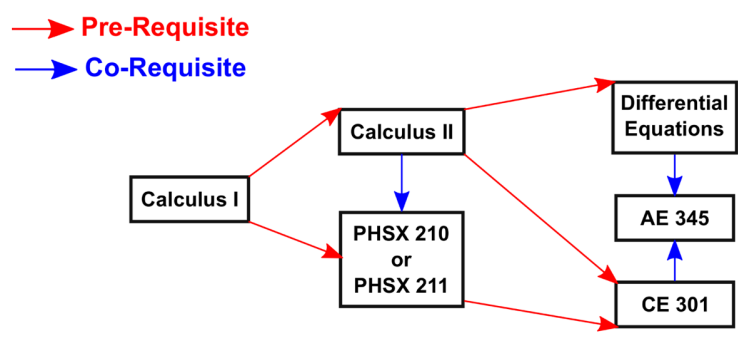

AE 345: Fluid Dynamics

FIG. 9. The prerequisites and corequisites for C\&PE 221 (Chemical Engineering Thermodynamics) and AE 345 (Fluid Dynamics).

301 or a course in differential equations as a corequisite (Fig. 9). Since General Physics I is not a prerequisite for $\mathrm{AE}$ 345, we would expect General Physics I enrollment would have little (direct) impact on student performance in $\mathrm{AE}$ 345; indeed, we can consider AE 345 to constitute a "control" for our analysis. However, since the content of PHSX 211 for students in this analysis did not include fluid statics or dynamics, whereas the content of PHSX $210 \mathrm{did}$ (Table I), it would not be surprising if the PHSX 210 cohort outperformed the PHSX 211 cohort in AE 345.

As was done previously, we calculated predicted distributions for students earning an A or a B in C\&PE 221

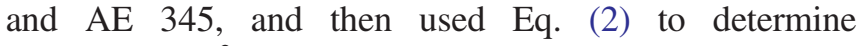
the summed $\chi^{2}$ values for students earning these grades. As shown in Table III, prior enrollment in PHSX 211 resulted in lower predictive power of ACT math scores on student grades in both C\&PE 221 and AE 345.

TABLE III. The summed $\chi^{2}$ values calculated using Eq. (2) for students earning an $\mathrm{A}$ or $\mathrm{B}$ in the listed course from the fall semester of 2015 through the spring semester of 2018 .

\begin{tabular}{lccccc}
\hline \hline & \multicolumn{2}{c}{ Sample size } & & \multicolumn{2}{c}{$\chi^{2}$} \\
\cline { 2 - 3 } \cline { 5 - 6 } Course & PHSX 210 & PHSX 211 & & PHSX 210 & PHSX 211 \\
\hline AE 345 & 71 & 13 & & 18.8 & 8.5 \\
C\&PE 221 & 154 & 15 & & 22.6 & 10.0 \\
CE 301 & 145 & 27 & & 23.4 & 17.0 \\
ME 211 & 125 & 72 & & 33.0 & 22.6 \\
ME 312 & 179 & 60 & & 33.8 & 19.3 \\
PHSX 212 & 592 & 199 & & 42.4 & 41.3 \\
\hline \hline
\end{tabular}

\section{DISCUSSION AND FUTURE WORK}

In this study we assessed whether our new curriculum for calculus-based introductory physics [17] improved student learning, both in terms of a better conceptual understanding of course content and an improved ability to apply physics principles or methods when solving quantitative problems. The data presented here demonstrate that students completing our new curriculum display a larger average normalized change in FCI exam scores than students completing a traditional curriculum [18], suggesting that our new curriculum has improved the learning of the physics concepts assessed by the FCI. These data also indicate that completion of our new curriculum can improve the downstream course grades of students with lower ACT math scores, and thus are consistent with an improvement in the ability of those students to apply physics concepts in quantitative problem solving outside the physics classroom.

Although we believe that the results presented here are consistent with the conclusion that our new curriculum has improved student learning, both locally and in downstream courses, it is important to note the limitations of this work. This also leads naturally to a discussion of the future directions of our research.

\section{A. Data resolution}

Our ability to resolve differences in downstream course grades earned by the PHSX 210 and PHSX 211 cohorts naturally depends upon the grade distributions for these downstream courses. For example, as shown in Fig. 10, while the grades earned in ME 211 from the fall semester of 2015 through the spring semester of 2018 are well described by a normal distribution (with a mean of B), the grades earned in AE 345 and PHSX 212 during this same time period are not; the grades earned in C\&PE 221, CE 301, and ME 312 are also well described by normal distributions with means around B (data not shown). It is therefore more difficult to impact the distributions of grades earned in both AE 345 and PHSX 212 since most students earn an A in those courses. While this may account for the similarity in the summed $\chi^{2}$ values for PHSX 212, it also makes the difference in the summed $\chi^{2}$ values for $\mathrm{AE}$ 345 more dramatic. The effectiveness of our $\chi^{2}$ analysis as a metric of student learning and performance is further reduced by the fact that we lose resolution in the data by discretizing course grades into only five categories (A, B, C, D, and F) and by the likelihood of grade curving occurring in these downstream courses, especially if those curves are nonlinear transformations.

\section{B. Possible causes of improved student performance}

The smaller class size of PHSX 211 could contribute to the better student learning of course content in PHSX 211; the enrollment in PHSX 211 is less than half the enrollment in PHSX 210. Effects due to class size are mitigated to some 


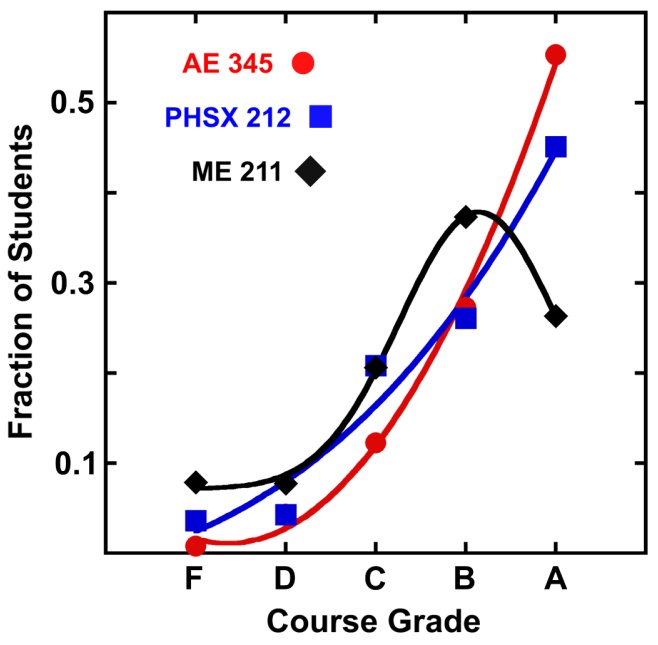

FIG. 10. The fraction of total enrollment in AE 345 (red circles), PHSX 212 (blue squares), and ME 211 (black diamonds) earning each letter grade from the fall semester of 2015 through the spring semester of 2018. The solid lines are generated from fits of these data to normal distributions and are meant to guide the eye.

degree, however, since the ratio of undergraduate teaching assistants to students is the same in PHSX 210 and PHSX 211. Similarly, while the content delivery of PHSX 210 and PHSX 211 are the same (both courses employ the same student-centered active learning approaches), PHSX 211 does include an extra 25 min of class time twice a week. It is possible that this extra time results in improvements in student learning. We plan to assess the impact of differences in class size and contact hours in a future study by teaching two different sections of PHSX 210, one with our new curriculum [17] and one with the traditional curriculum [18], and repeating the FCI exam and downstream course grade analysis presented here for these two sections.

We propose that the observed improvements in student performance in downstream courses correlated with completion of our new curriculum (Table III) result from a combination of improved learning of physics content and improved applied mathematics skills. For example, allowing students to develop their conceptual and computational skills for classical mechanics before introducing forces may enable students to focus more effort on learning or practicing the vector mathematics necessary for correctly applying Newton's laws. Since the majority of course content in ME 211 (Statics and Dynamics) and CE 301 (Statics and Dynamics) consists of static equilibrium problems for which force-based solutions are required, the improvement in student performance in those courses may reflect increased student proficiency in the application of forces and Newton's laws to solve problems. Similarly, the calculus-enhanced presentation of thermodynamics in our new curriculum likely better prepares students for success in ME 312 (Basic Engineering Thermodynamics) and C\&PE 221 (Chemical Engineering Thermodynamics) than the traditional approach to the topic. Additional work is clearly needed to assess the validity of both of these speculations.

The correlation between mathematics ability and performance in physics and engineering courses is often attributed to the emphasis in these courses on applied mathematics [27-33]. Since the two different cohorts of students in this study are otherwise completing the same curriculum of calculus courses, and completing these calculus courses with one another at the same time, the only difference in their mathematics preparation lies in whether they took PHSX 210 or PHSX 211. We therefore speculate that the reduction in the dependence of downstream course grades on ACT math scores may be associated with an improvement in students' applied mathematics skills resulting from the more calculus-intensive curriculum of PHSX 211 (Table I). Indeed, an improved proficiency with applied mathematics seems a more likely explanation for the improved student performance in $\mathrm{AE} 345$ rather than an improvement in physics concept knowledge since the content of PHSX 211 for students in this analysis did not include fluid statics or dynamics, whereas the content of PHSX 210 did. We intend to investigate the possibility that completion of our new curriculum has increased student proficiency with applied mathematics by measuring the normalized change on a math transference assessment given to students in these courses; we will either develop our own assessment or use existing assessments, if and applicable one is available.

\section{CONCLUSION}

The data presented here demonstrate that our calculusenhanced energy-first curriculum results in improved student learning of classical mechanics concepts and improved ability to apply physics principles or methods in downstream physics and engineering courses. Taken together, these data suggest that the improvements in student learning associated with our new curriculum may result from positive feedback between developing proficiency with physics concepts and increasing fluency with applied mathematics. In this way, our new curriculum may be lowering the math transference barrier to learning physics [10-13] by establishing an environment more conducive to learning and practicing both mathematics and physics.

As applied mathematics becomes an increasingly important component of all STEM disciplines [27,2933 ], there is a clear need to promote student competency with mathematical and computational skills across all STEM curricula. Introductory physics courses are the ideal venue to improve students' mathematics abilities as they emphasize the development of students' quantitative problem solving skills [34]. The results presented here demonstrate that student fluency with applied mathematics (vector mathematics and calculus) may be improved by using a calculus-enhanced energy-first curriculum [17] in 
introductory physics. We also note that since this curriculum can be implemented independently of established successful approaches of content delivery [2-4], it can complement these approaches rather than compete with them. However, this modification in content may be easier to implement than some modifications in content delivery as not all institutions have available the modified classroom space necessary for inquiry-based instruction $[7,8]$ or resources for successful implementation of peer instruction $5,6]]$. Furthermore, since the benefits of this new curriculum are largest for students with initially poorly mathematics ability, we believe that this curriculum has specific potential to benefit students with weaker secondary mathematics preparation, such as traditionally underserved populations [35,36], who are also at a higher risk of dropping out of these programs [31-33]. Finally, since introductory physics courses often satisfy universities' general education requirements as well as being required by nearly all STEM degree programs, improvements in the pedagogy of these courses can have benefits spanning many disciplines and career paths; for example, we demonstrate here that increasing the amount of applied mathematics content in introductory physics may be associated with improvements in student performance in downstream engineering courses. This in turn can lead to a more scientifically and mathematically literate populace, both inside and outside of STEM.

\section{ACKNOWLEDGMENTS}

We would like to thank Professor Alice Bean, Professor Allison Kirkpatrick, and Professor Graham Wilson for conversations that were useful in the writing of this manuscript. Analysis of aggregate student grades in downstream engineering courses was performed in collaboration with the University of Kansas STEM Analytics Program, which is supported by the American Association of Universities Undergraduate STEM Education Initiative funded by the Northrop Grumman Foundation. We thank Professor Andrea Greenhoot, Professor Doug Ward, and Dr. Trinia Ramirez for their help with this analysis. We also thank Professor Phil Baringer, Professor Stephen Sanders, and Professor Judy Wu, who together with Professor Michael Murray, taught PHSX 210 and PHSX 211 over the course of this study. The article processing charges related to the publication of this article were supported by The University of Kansas (KU) One University Open Access Author Fund sponsored jointly by the KU Provost, KU Vice Chancellor for Research \& Graduate Studies, and KUMC Vice Chancellor for Research and managed jointly by the Libraries at the Medical Center and KU - Lawrence.

\section{APPENDIX}

A system consists of a sphere with mass $m$ and radius $R$ rolling without slipping down the ramp shown in Fig. 11. What is the acceleration of the sphere if the system is

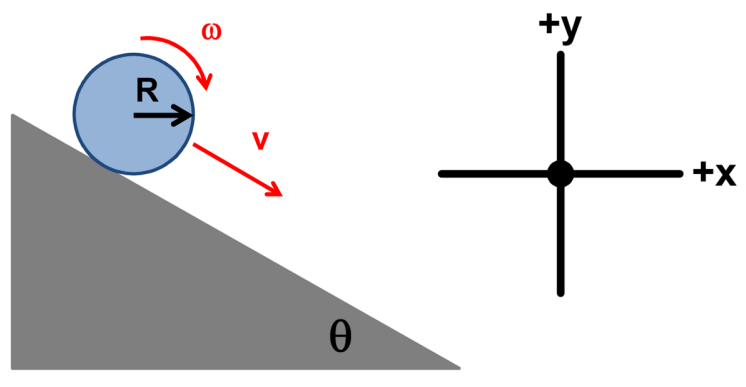

FIG. 11. A sphere with radius $R$ is rolling without slipping down a ramp. The coordinate system defines an $x$ axis parallel to the horizontal surface of the ramp and a $y$ axis parallel to the vertical surface of the ramp. The positive direction for the $x$ axis points to the right and the positive direction for the $y$ axis points upwards. The angle of the ramp with respect to the $x$ axis is $\theta$.

isolated? A coordinate system for the problem in which the $x$ axis is parallel to the horizontal surface of the ramp and the $y$ axis is parallel to the vertical surface of the ramp is also indicated in Fig. 11. The positive direction for the $x$ axis points to the right in Fig. 11 and the positive direction for the $y$ axis points upwards in Fig. 11 .

\section{Force-first solution}

We begin by drawing the free-body diagram for the sphere. As shown in Fig. 12, we can define an $s$ axis to be parallel to the ramp's surface with a positive direction pointing down the ramp. In addition to including the force of gravity $\left(F_{g}\right)$ and the normal force $(n)$, the correct drawing of the free body diagram requires us to know a priori to include the force of static friction $\left(F_{s}\right)$. Applying Newton's 2nd law to the $s$ axis in Fig. 12 gives us the following equation:

$$
\left(F_{\text {net }}\right)_{s}=m a_{s} \rightarrow F_{g} \sin \theta-F_{s}=m a_{s} .
$$

The subscript $s$ denotes that the acceleration is along the $s$ axis (parallel to the ramp's surface). Next we must calculate

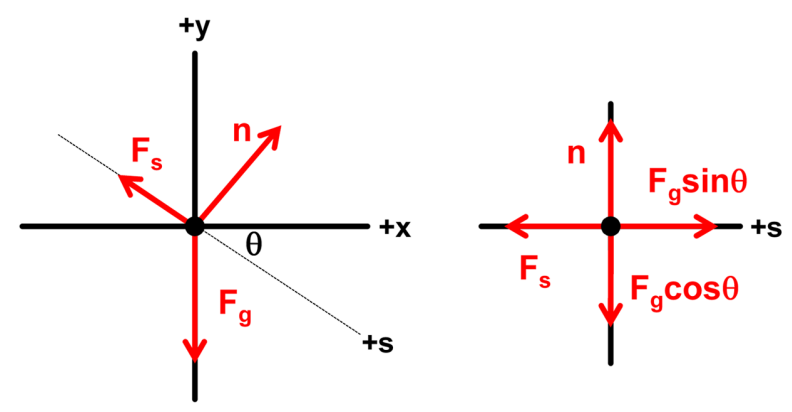

FIG. 12. The free-body diagrams describing the forces acting on the rolling sphere in Fig. 11. The positive direction for the $s$ axis points down the ramp. The variables $F_{g}, F_{s}$, and $n$ denote the force of gravity, the static friction force, and the normal force, respectively. The angle $\theta$ is as indicated in Fig. 11. 
the net torque acting on the sphere. Of the forces shown in Fig. 12, only $F_{s}$ is associated with a nonzero torque. Thus, applying Newton's 2nd law for the net torque gives us the following equation relating $F_{s}$ to the sphere's moment of inertia $(I)$ and angular acceleration $(\alpha)$ :

$$
\tau_{\text {net }}=I \alpha \rightarrow F_{s} \sin \left(\frac{3 \pi}{2}\right)=I \alpha \rightarrow F_{s}=-\left(\frac{I}{R}\right) \alpha .
$$

We can then substitute this result into the net force equation.

$$
F_{g} \sin \theta-\left[-\left(\frac{I}{R}\right) \alpha\right]=m a_{s} \rightarrow F_{g} \sin \theta+\left(\frac{I}{R}\right) \alpha=m a_{s} .
$$

Since the sphere rolls without slipping, the tangential and angular speeds are related. In this particular case, we note that a positive direction of motion along the $s$ axis is associated with a clockwise rotation of the sphere. Therefore, a positive value for $v_{s}$ (the velocity along the $s$ axis) corresponds to a negative value of $\omega$. Taking the derivative of this relationship with respect to time gives us the following expression relating the tangential and angular acceleration:

$$
\omega=-\frac{v_{s}}{R} \rightarrow \alpha=-\frac{a_{s}}{R} .
$$

Substitution of this result and the expression for the magnitude of the force of gravity $\left(F_{g}=m g\right)$ then gives us the following:

$$
\begin{aligned}
m g \sin \theta+\left(\frac{I}{R}\right)\left(-\frac{a_{s}}{R}\right) & =m a_{s} \rightarrow m g \sin \theta=\left(m+\frac{I}{R^{2}}\right) a_{s} \\
a_{s} & =\left(\frac{m R^{2}}{I+m R^{2}}\right) g \sin \theta .
\end{aligned}
$$

The positive sign in the solution for the $a_{s}$ indicates that the direction of the acceleration is along the positive direction of the $s$ axis in Fig. 12. In other words, the acceleration is directed down the ramp.

\section{Energy-first solution}

We begin by writing the equation for the total energy of the system, which consists of translational kinetic energy $(K)$, rotational kinetic energy $\left(K_{\text {rot }}\right)$, and gravitational potential energy $\left(U_{g}\right)$ :

$$
E=K+K_{\mathrm{rot}}+U_{g} \rightarrow E=\frac{1}{2} m v_{s}^{2}+\frac{1}{2} I \omega^{2}+m g y .
$$

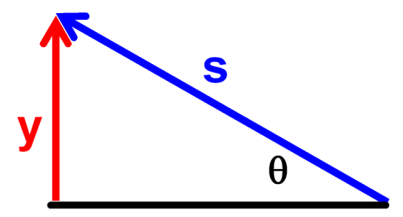

FIG. 13. The relationship between the variables $y$ and $s$ for denoting the position of the rolling sphere in Fig. 11.

The subscript $s$ denotes that the translational speed is along the $s$ axis (parallel to the ramp's surface). We can relate motion along the vertical $y$ axis to motion along the $s$ axis using trigonometry, as shown in Fig. 13:

$$
y=s(\sin \theta) \rightarrow E=\frac{1}{2} m v_{s}^{2}+\frac{1}{2} I \omega^{2}+m g s(\sin \theta) .
$$

Since the sphere rolls without slipping, the tangential and angular speeds are related.

$$
\begin{gathered}
\omega= \pm \frac{v_{s}}{R} \rightarrow E=\frac{1}{2} m v_{s}^{2}+\frac{1}{2} I\left( \pm \frac{v_{s}}{R}\right)^{2}+m g s(\sin \theta) \\
E=\frac{1}{2}\left(m+\frac{I}{R^{2}}\right) v_{s}^{2}+m g s(\sin \theta) .
\end{gathered}
$$

Since the system is isolated, its energy is conserved (i.e., energy is constant). Thus, the first derivatives of energy are zero. Taking the derivative with respect to $s$ gives us

$$
\begin{aligned}
\frac{d E}{d s} & =0 \rightarrow\left(m+\frac{I}{R^{2}}\right) a_{s}+m g(\sin \theta)=0 \\
a_{s} & =-\left(\frac{m R^{2}}{I+m R^{2}}\right) g \sin \theta .
\end{aligned}
$$

The negative sign in the solution for the $a_{s}$ indicates that the direction of the acceleration is along the negative direction of the $s$ axis in Fig. 13. In other words, the acceleration is directed down the ramp. This value for $a_{s}$, magnitude and direction, is identical to what was derived above using a force-based solution. We argue that the energy-first solution is more tractable for students, however, since it (i) is computationally simpler, (ii) does not require students to incorporate the static friction force, which can be overlooked easily, (iii) allows them to avoid errors with inconsistent use of positive and negative signs denoting the direction of forces and torques or relating angular and tangential velocity and acceleration, and (iv) follows a problem-solving strategy identical to that they use to solve other problems in the course and in downstream courses in physics and other STEM disciplines. 
[1] T. D. Snyder, C. de Brey, and S. A. Dillow, Digest of Education Statistics 2015, National Center for Education Statistics, Tech. Rep. (2015), https://eric.ed.gov/? id=ED565675.

[2] R. R. Hake, Interactive-engagement vs traditional methods: A six-thousand-student survey of mechanics test data for introductory physics courses, Am. J. Phys. 66, 64 (1998).

[3] S. Freeman, S. L. Eddy, M. McDonough, M. K. Smith, N. Okoroafor, H. Jordt, and M. P. Wenderoth, Active learning increases student performance in science, engineering, and mathematics, Proc. Natl. Acad. Sci. U.S.A. 111, 8410 (2014).

[4] H. L. Iverson, D. C. Briggs, M. A. Ruiz-Primo, R. M. Talbot, L. A. Shepard, M. Sabella, C. Henderson, and C. Singh, Undergraduate physics course innovations and their impact on student learning, AIP Conf. Proc. 1179, 169 (2009).

[5] C. H. Crouch and E. Mazur, Peer Instruction: Ten years of experience and results, Am. J. Phys. 69, 970 (2001).

[6] A. P. Fagen, C. H. Crouch, and E. Mazur, Peer instruction: Results from a range of classrooms, Phys. Teach. 40, 206 (2002).

[7] E. L. Chiappetta and A. D. Adams, Inquiry-based instruction, Sci. Teach. 71, 46 (2004).

[8] D. D. Minner, A. Jurist Levy, and J. Century, Inquiry-based science instruction-what is it and does it matter? Results from a research synthesis years 1984 to 2002, J. Res. Sci. Teach. 47, 474 (2010).

[9] N. S. Rebello, L. Cui, A. G. Bennett, D. A. Zollman, and D. J. Ozimek, Transfer of learning in problem solving in the context of mathematics and physics, in Learning to Solve Complex Scientific Problems (Lawrence Earlbaum, Mahwah, NJ, 2007), pp. 223-246.

[10] R. D. Knight, The vector knowledge of beginning physics students, Phys. Teach. 33, 74 (1995).

[11] N.-L. Nguyen and D. E. Meltzer, Initial understanding of vector concepts among students in introductory physics courses, Am. J. Phys. 71, 630 (2003).

[12] D. J. Ozimek, Retention and transfer from trigonometry to physics, AIP Conf. Proc. 790, 173 (2005).

[13] B. D. Mikula and A. F. Heckler, Student difficulties with trigonometric vector components persist in multiple student populations, in Proceedings of the 2013 Physics Education Research Conference, Portland, OR, edited by P. V. Engelhardt, A. D. Churukian, and D. L. Jones (AIP, New York, 2014), pp. 253-256.

[14] A. B. Champagne, L. E. Klopfer, and J. H. Anderson, Factors influencing the learning of classical mechanics, Am. J. Phys. 48, 1074 (1979).

[15] F. Goldberg, V. Otero, and S. Robertson, Design principles for effective physics instruction: A case from physics and everyday thinking, Am. J. Phys. 78, 1265 (2010).

[16] D. Hestenes, M. Wells, and G. Swackhamer, Force concept inventory, Phys. Teach. 30, 141 (1992).

[17] C. J. Fischer, The energy of physics, Part I: Classical Mechanics and Thermodynamics (Cognella, Inc., San Diego, CA, 2015).
[18] R. A. Serway and J. W. Jewett, Physics for Scientists and Engineers with Modern Physics (Cengage Learning, Boston, MA, 2018).

[19] https://www.physport.org/.

[20] J. D. Marx and K. Cummings, Normalized change, Am. J. Phys. 75, 87 (2007).

[21] R. R. Hake, R. Wakeland, A. Bhattacharyya, and R. Sirochman, Assessment of individual student performance in an introductory mechanics course, AAPT Announcer 24, 76 (1994).

[22] M. J. Thoresen and C. M. Gross, Factors contributing to success in college physics, AAPT Announcer 30, 128 (2000).

[23] D. E. Meltzer, The relationship between mathematics preparation and conceptual learning gains in physics: A possible hidden variable in diagnostic pretest scores, Am. J. Phys. 70, 1259 (2002).

[24] J. J. Pembridge and M. A. Verleger, First-year math and physics courses and their role in predicting academic success in subsequent courses, in 120nd American Society for Engineering Education (ASEE) Annual Conference \& Exposition (American Society for Engineering Education, Washington, DC, 2013).

[25] J. R. Taylor, Error Analysis (Univ. Science Books, Sausalito, CA, 1997).

[26] E. M. Pugh and G. H. Winslow, The Analysis of Physical Measurements (Addison-Wesley, Reading, MA, 1966), http://cds.cern.ch/record/102988.

[27] H. T. Hudson and W. R. McIntire, Correlation between mathematical skills and success in physics, Am. J. Phys. 45, 470 (1977).

[28] H. Daniel Cohen, D. F. Hillman, and R. M. Agne, Cognitive level and college physics achievement, Am. J. Phys. 46, 1026 (1978).

[29] H. T. Hudson and R. M. Rottmann, Correlation between performance in physics and prior mathematics knowledge, J. Res. Sci. Teach. 18, 291 (1981).

[30] S. E. Brekke, Some factors affecting student performance in physics (1994), https://eric.ed.gov/?id=ED390650.

[31] C. Moller-Wong and A. Eide, An engineering student retention study, J. Engin. Educ. 86, 7 (1997).

[32] W. Tyson, R. Lee, K. M. Borman, and M. Ann Hanson, Science, technology, engineering, and mathematics (STEM) pathways: High school science and math coursework and postsecondary degree attainment, J. Educ. Students Placed at Risk (JESPAR) 12, 243 (2007).

[33] G. Zhang, T. J. Anderson, M. W. Ohland, and B. R. Thorndyke, Identifying factors influencing engineering student graduation: A longitudinal and cross-institutional study, J. Engin. Educ. 93, 313 (2004).

[34] E. F. Redish, Problem solving and the use of math in physics courses, arXiv:physics/0608268.

[35] R. Tsoi-A and F. Bryant, College preparation for African American students: Gaps in the high school educational experience (2015), https://vtechworks.lib.vt.edu/handle/ $10919 / 83649$.

[36] F. Atuahene and T. A. Russell, Mathematics readiness of first-year university students, J. Develop. Educ. 39, 12 (2016). 